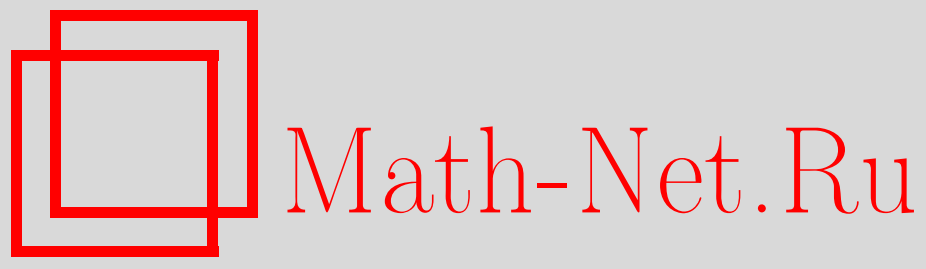

О. С. Якимова, Индекс централизаторов элементов в классических алгебрах Ли, Функи. анализ и его прил., 2006, том 40, выпуск 1, 52-64

DOI: https://doi.org/10.4213/faa18

Использование Общероссийского математического портала Math-Net.Ru подразумевает, что вы прочитали и согласны с пользовательским соглашением

http://www . mathnet.ru/rus/agreement

Параметры загрузки:

IP : 54.157 .27 .8

26 апреля 2023 г., 13:14:34

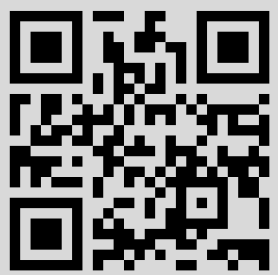




\title{
Индекс централизаторов элементов в классических алгебрах Ли*
}

\author{
(c) 2006. О. С. ЯкимовА
}

\section{Введение}

Пусть $\mathfrak{g}$ - алгебра Ли над полем $\mathbb{K}$. Рассмотрим коприсоединенное действие $\operatorname{ad}^{*}(\mathfrak{g})$. Индекс алгебры Ли $\mathfrak{g}$ - это минимум размерностей стабилизаторов $\mathfrak{g}_{\alpha}$ по всем ковекторам $\alpha \in \mathfrak{g}^{*}$,

$$
\operatorname{index} \mathfrak{g}=\min _{\alpha \in \mathfrak{g}^{*}} \operatorname{dim} \mathfrak{g}_{\alpha} .
$$

Определение индекса было дано Диксмье в $[2,11.1 .6]$. Это понятие играет важную роль в теории представлений, а также в теории инвариантов. Согласно теореме Розенлихта [12], орбиты общего положения произвольного действия линейной алгебраической группы на неприводимом алгебраическом многообразии разделяются рациональными инвариантами; в частности, index $\mathfrak{g}=\operatorname{tr} . \operatorname{deg} \mathbb{K}\left(\mathfrak{g}^{*}\right)^{G}$

Индекс редуктивной алгебры равен ее рангу. Задача нахождения индекса произвольной алгебры Ли является, по-видимому, необозримой. Однако для некоторых классов нередуктивных подалгебр редуктивных алгебр Ли получен ряд интересных результатов. Так, в работах $[3,8,13]$ рассматриваются параболические и связанные с ними подалгебры (нильпотентные радикалы, алгебры водорослевого типа). Другой интересный класс подалгебр - это централизаторы элементов. Для них задача нахождения индекса интересна тем, что имеет непосредственное отношение к теории интегрируемых гамильтоновых систем.

Пусть $G$ - полупростая комплексная или вещественная группа Ли, $\mathfrak{g}=\operatorname{Lie} G$ и $G x$ - орбита некоторого ковектора $x \in \mathfrak{g}^{*}$. Обозначим стабилизатор точки $x$ через $\mathfrak{g}_{x}$. Хорошо известно, что орбита $G x$ обладает $G$-инвариантной симплектической структурой. Равенство index $\mathfrak{g}_{x}=$ index $\mathfrak{g}$ равносильно существованию полученного методом сдвига аргумента семейства коммутирующих относительно скобки Пуассона полиномиальных функций на $\mathfrak{g}^{*}$, ограничение которого на $G x$ содержит $\frac{1}{2} \operatorname{dim}(G x)$ алгебраически независимых функций [1].

ГиПотезА (Элашвили). Пусть $\mathfrak{g}-$ редуктивная алгебра Ли. Тогда index $\mathfrak{g}_{x}=$ index $\mathfrak{g}$ для любого ковектора $x \in \mathfrak{g}^{*}$.

Напомним, что в случае редуктивной алгебры $\mathfrak{g}$ имеет место изоморфизм $\mathfrak{g}$-модулей $\mathfrak{g}^{*} \cong \mathfrak{g}$. В частности, достаточно доказать «гипотезу об индексе» для стабилизаторов $\mathfrak{g}_{x}$ векторов $x \in \mathfrak{g}$.

Пусть $x \in \mathfrak{g}$ и $x=x_{s}+x_{n}$ - разложение Жордана этого элемента. Тогда $\mathfrak{g}_{x}=$ $\left(\mathfrak{g}_{x_{s}}\right)_{x_{n}}$. Подалгебра $\mathfrak{g}_{x_{s}}$ редуктивна и содержит максимальный тор алгебры $\mathfrak{g}$. Следовательно, index $\mathfrak{g}_{x_{s}}=$ index $\mathfrak{g}=\mathrm{rk} \mathfrak{g}$. Таким образом, проверка «гипотезы

* Работа выполнена при поддержке гранта CRDF RM1-2543-MO-03 и гранта РФФИ №0501-00988. 
об индексе» сводится к вычислению индексов стабилизаторов нильпотентных элементов. Легко видеть, что можно ограничиться случаем простой алгебры $\mathfrak{g}$.

Отметим, что если $x$ - регулярный нильпотент, то его стабилизатор $\mathfrak{g}_{x}$ коммутативен и имеет размерность rk g. Ранее «гипотеза об индексе» была доказана для субрегулярных нильпотентов и нильпотентов высоты 2 в [9], а также для нильпотентов высоты 3 в [10]. (Высотой нильпотента $e$ называется наибольшее число $m$, такое, что $(\operatorname{ad} e)^{m} \neq 0$.)

В настоящей работе доказано, что индекс централизатора $\mathfrak{z}(e):=\mathfrak{g}_{e}$ нильпотентного элемента $e \in \mathfrak{g}$ простой классической алгебры Ли равен рангу алгебры Ли $\mathfrak{g}$. Заметим, что если редуктивная алгебра $\mathfrak{g}$ не содержит исключительных идеалов, то и алгебра $\mathfrak{g}_{x_{s}}$ обладает этим свойством. Таким образом, гипотеза об индексе доказана для прямых сумм классических алгебр.

Согласно неравенству Винберга, подробно изложенному в [9, Sec. 1], index $\mathfrak{g}_{x} \geqslant$ index $\mathfrak{g}$ для любого элемента $x \in \mathfrak{g}^{*}$. Остается получить оценку в другую сторону, для чего достаточно предъявить такую точку $\alpha \in\left(\mathfrak{g}_{x}\right)^{*}$, что размерность ее стабилизатора в алгебре $\mathfrak{g}_{x}$ не превосходит $\mathrm{rk} \mathfrak{g}$. Для алгебр $\mathfrak{g}=\mathfrak{g l}(V)$ и $\mathfrak{g}=\mathfrak{s p}(V)$ явно указана такая точка $\alpha \in \mathfrak{g}_{e}^{*}$. В случае ортогональной алгебры часть доказательства проведена по индукции. Предполагается, что основное поле $\mathbb{K}$ содержит как минимум $k$ элементов, где $k$ - число жордановых клеток нильпотента $e \in \mathfrak{g}$. В случаях ортогональной и симплектической алгебры предполагается также, что характеристика основного поля не равна 2.

В последних двух параграфах поле $\mathbb{K}$ алгебраически замкнуто и его характеристика равна 0. Показано, что построенные в случаях $\mathfrak{g}=\mathfrak{g l}(V)$ и $\mathfrak{g}=\mathfrak{s p}(V)$ стабилизаторы $\left(\mathfrak{g}_{e}\right)_{\alpha}$ являются стабилизаторами общего положения коприсоединенного представления алгебры $\mathfrak{g}_{e}$. В ортогональном случае приведен пример нильпотента $e \in \mathfrak{s o}_{8}$, для которого коприсоединенное действие алгебры $\mathfrak{g}_{e}$ не имеет стабилизатора общего положения. Аналогичные результаты для параболических подалгебр и подалгебр водорослевого типа простых алгебр Ли получены Панюшевым, а также Товелем и Ю. В работе [13] построен пример параболической подалгебры в $\mathfrak{s o}_{8}$, коприсоединенное действие которой не имеет стабилизатора общего положения. Для серий $A$ и $C$ существование стабилизатора общего положения доказано Панюшевым [11].

В заключительной части работы рассматривается коммутаторное многообразие алгебры $\mathfrak{g}_{e}$ и его связь с коммутаторным многообразием троек матриц.

Когда данная работа уже была принята к печати, автору стало известно, что гипотеза Элашвили доказана в статье Шарбоннеля [5] для случая $\mathbb{K}=\mathbb{C}$.

\section{§1. Предварительные сведения}

Пусть $\mathfrak{g}$ - простая классическая алгебра Ли или полная линейная алгебра. Пусть $e \in \mathfrak{g}$ - нильпотентный элемент и $\mathfrak{z}(e)$ - его централизатор в $\mathfrak{g}$. Заметим, что случай $\mathfrak{g}=\mathfrak{g l}(V)$ ничем принципиально не отличается от случая $\mathfrak{g}=\mathfrak{s l}(V)$. Однако он удобнее для вычислений. В случаях ортогональной и симплектической алгебр нам понадобятся некоторые факты из теории симметрических пространств.

Пусть $(,)_{V}$ - невырожденная симметрическая или кососимметрическая форма на конечномерном линейном пространстве $V$, заданная матрицей $J$, т. е. $(v, w)_{V}=v^{t} J w$, где знак ${ }^{t}$ означает транспонирование. Элементы алгебры $\mathfrak{g l}(V)$, сохраняющие форму $(,)_{V}$, составляют в точности множество $\mathfrak{g l}(V)^{\sigma}$ 
неподвижных точек инволюции $\sigma(\xi)=-J \xi^{t} J^{-1}$. Имеет место $\mathfrak{g l}(V)^{\sigma}$-инвариантное разложение $\mathfrak{g l}(V)=\mathfrak{g l}(V)^{\sigma} \oplus \mathfrak{g}_{1}$, причем элементы подпространства $\mathfrak{g}_{1}$ умножают форму $(,)_{V}$ на -1 , т. е. $(\xi v, w)_{V}=(v, \xi w)_{V}$ для любых $v, w \in V$.

Пусть $\mathfrak{g}=\mathfrak{g l}(V)^{\sigma}$ и $e \in \mathfrak{g}$ - нильпотентный элемент. Обозначим его централизаторы в алгебрах $\mathfrak{g}$ и $\mathfrak{g l}(V)$ через $\mathfrak{z}(e)$ и $\mathfrak{z} \mathfrak{g l}(e)$ соответственно. Поскольку $\sigma(e)=e$, инволюция $\sigma$ действует на централизаторе $\mathfrak{z g r}(e)$. Легко видеть, что $\mathfrak{z}_{\mathfrak{g l}}(e)^{\sigma}=\mathfrak{z}(e)$. Возникает $\mathfrak{z}(e)$-инвариантное разложение $\mathfrak{z} \mathfrak{g l}(e)=\mathfrak{z}(e) \oplus \mathfrak{z} 1$. Пусть $\alpha \in \mathfrak{z}_{\mathfrak{g l}}(e)^{*}$ и $\tilde{\alpha}$ - ограничение точки $\alpha$ на $\mathfrak{z}(e)$.

ПРЕДЛОЖЕНИЕ 1. Пустъ $\alpha \in \mathfrak{z}_{\mathfrak{g l}}(e)^{*} u \alpha\left(\mathfrak{z}_{1}\right)=0$. Тогда $\mathfrak{z}(e)_{\tilde{\alpha}}=\mathfrak{z}_{\mathfrak{g l}}(e)_{\alpha} \cap \mathfrak{z}(e)$.

Доказательство. Пусть $\xi \in \mathfrak{z}(e)$. Так как $\left[\xi, \mathfrak{z}_{1}\right] \subset \mathfrak{z}_{1}$, то $\alpha([\xi, \mathfrak{z}(e)])=0$ тогда и только тогда, когда $\alpha([\xi, \mathfrak{z} \mathfrak{g l}(e)])=0$. В частности, $\mathfrak{z}(e)_{\tilde{\alpha}}=\mathfrak{z}(e)_{\alpha}$.

Пусть $\mathfrak{h}$ - алгебра Ли и $\tau \in$ Auth - инволюция, задающая разложение $\mathfrak{h}=\mathfrak{h}_{0} \oplus \mathfrak{h}_{1}$. Каждая точка $\gamma \in \mathfrak{h}_{0}^{*}$ определяет кососимметрическую 2-форму $\hat{\gamma}$ на $\mathfrak{h}_{1}$ по формуле $\hat{\gamma}(\xi, \eta)=\gamma([\xi, \eta])$.

ЛЕмма 1. В описанных выше обозначениях

$$
\operatorname{index} \mathfrak{h} \leqslant \operatorname{index} \mathfrak{h}_{0}+\min _{\gamma \in \mathfrak{h}_{0}^{*}} \operatorname{dim}(\operatorname{Ker} \hat{\gamma})
$$

ДокАЗАтельство. Рассмотрим точку $\gamma$ как функцию на $\mathfrak{h}$, равную нулю на $\mathfrak{h}_{1}$. Тогда $\mathfrak{h}_{\gamma}=\left(\mathfrak{h}_{0}\right)_{\gamma} \oplus\left(\mathfrak{h}_{\gamma} \cap \mathfrak{h}_{1}\right)=\left(\mathfrak{h}_{0}\right)_{\gamma} \oplus(\operatorname{Ker} \hat{\gamma})$. Для точек общего положения (принадлежащих открытому по Зарисскому подмножеству $\left.U_{1} \subset \mathfrak{h}_{0}^{*}\right) \operatorname{dim}\left(\mathfrak{h}_{0}\right)_{\gamma}=$ index $\mathfrak{h}_{0}$. Для точек другого открытого подмножества $U_{2} \subset \mathfrak{h}_{0}^{*}$ пространство $\operatorname{Ker} \hat{\gamma}$ имеет минимально возможную размерность. Для элементов пересечения $U_{1} \cap U_{2}$ размерность стабилизатора в $\mathfrak{h}$ совпадает с указанной в лемме суммой.

\section{§2. Полная линейная алгебра}

Рассмотрим нильпотентный элемент $e \in \mathfrak{g l}(V)$, где $V$ есть $n$-мерное линейное пространство над полем $\mathbb{K}$. Его централизатор обозначим через $\mathfrak{z}(e)$. Покажем, что индекс алгебры $\mathfrak{z}(e)$ равен $n$.

Пусть $k$ - число жордановых клеток элемента $e$ и $W \subset V$ - некоторое дополнительное подпространство к Im $e$ размерности $k$. Обозначим через $d_{i}+1$ размер $i$-й жордановой клетки. Выберем в пространстве $W$ такой базис $w_{1}, \ldots, w_{k}$, что $e^{d_{i}+1} w_{i}=0$ для каждого $i$, а векторы $e^{s} w_{i}$ при $1 \leqslant i \leqslant k, 0 \leqslant s \leqslant d_{i}$ образуют базис пространства $V$. Будем называть векторы $w_{i}$ образующими жсордановых клеток, а пространства $V[i]=\left\langle w_{i}, e w_{i}, \ldots, e^{d_{i}} w_{i}\right\rangle$ пространствами жсордановых клеток. Будем говорить, что набору жордановых клеток с номерами $i_{1}, \ldots, i_{t}$ соответствует подпространство $V\left[i_{1}\right] \oplus \cdots \oplus V\left[i_{t}\right]$. Пусть $\varphi \in \mathfrak{z}(e)$. Поскольку $\varphi\left(e^{s} w_{i}\right)=e^{s} \varphi\left(w_{i}\right)$, элемент $\varphi$ полностью определяется своими значениями на векторах $w_{i}$. Каждое такое значение имеет вид

$$
\varphi\left(w_{i}\right)=\sum_{j, s} c_{i}^{j, s} e^{s} w_{j}, \quad \text { где } c_{i}^{j, s} \in \mathbb{K} .
$$

Таким образом, отображение $\varphi$ полностью определяется своими коэффициентами $c_{i}^{j, s}=c_{i}^{j, s}(\varphi)$. Элемент $\varphi \in \mathfrak{z}(e)$ сохраняет пространства жордановых клеток тогда и только тогда, когда $c_{i}^{j, s}(\varphi)=0$ при $i \neq j$. 
В алгебре $\mathfrak{z}(e)$ можно выбрать базис, состоящий из элементов $\xi_{i}^{j, s}$, таких, что

$$
\xi_{i}^{j, s}\left(w_{i}\right)=e^{s} w_{j}, \quad \xi_{i}^{j, s}\left(w_{t}\right)=0 \quad \text { при } t \neq i,
$$

где $d_{j}-d_{i} \leqslant s \leqslant d_{j}$ при $d_{j} \geqslant d_{i}$ и $0 \leqslant s \leqslant d_{i}$ при $d_{j}<d_{i}$.

Рассмотрим точку $\alpha \in \mathfrak{z}(e)^{*}$, заданную формулой

$$
\alpha(\varphi)=\sum_{i=1}^{k} a_{i} c_{i}^{i, d_{i}}, \quad a_{i} \in \mathbb{K}
$$

где $c_{i}^{j, s}-$ коэффициенты элемента $\varphi \in \mathfrak{z}(e)$ и $a_{i}$ - попарно различные ненулевые числа. При $i=j, s=d_{i}$ значение $\alpha\left(\xi_{i}^{j, s}\right)$ равно $a_{i}$; в противном случае $\alpha\left(\xi_{i}^{j, s}\right)$ равно нулю.

Tеорема 1. Стабилизатор $\mathfrak{z}(e)_{\alpha}$ точки $\alpha$ в алгебре $\mathfrak{z}(e)$ coстоит из преобразований, сохраняющих пространства эсордановых клеток, т. е. является линейной оболочкой элементов $\xi_{i}^{i, s}$.

ДокАЗАтельство. Пусть элемент $\varphi \in \mathfrak{z}(e)$ задан формулой $(1)$. (Некоторые из чисел $c_{i}^{j, s}$ должны быть равны нулю, но для нас это неважно.) Для каждого базисного вектора $\xi\left(w_{i}\right)=e^{b} w_{j}$ имеем

$$
\alpha\left(\left[\xi_{i}^{j, b}, \varphi\right]\right)=\alpha\left(\sum_{t, s} c_{t}^{i, s} \xi_{t}^{j, s+b}-\sum_{t, s} c_{j}^{t, s} \xi_{i}^{t, s+b}\right)=a_{j} c_{j}^{i, d_{j}-b}-a_{i} c_{j}^{i, d_{i}-b} .
$$

Элемент $\varphi$ лежит в стабилизаторе $\mathfrak{z}(e)_{\alpha}$ тогда и только тогда, когда $\alpha\left(\left[\varphi, \xi_{i}^{j, b}\right]\right)$ $=0$ для всех $\xi_{i}^{j, b}$.

Заметим, что если $\varphi$ сохраняет пространства жордановых клеток, т. е. $c_{i}^{j, s}=0$ при $i \neq j$, то $\alpha([\varphi, \mathfrak{z}(e)])=0$. Покажем, что никакие другие элементы не лежат в стабилизаторе точки $\alpha$. Пусть какое-то $c_{i}^{j, s}$ отлично от 0 и $i \neq j$. Возможны три различных случая: $d_{i}<d_{j}, d_{i}=d_{j}$ и $d_{i}>d_{j}$.

Если $d_{j} \leqslant d_{i}$, то положим $\xi\left(w_{j}\right)=e^{d_{i}-s} w_{i}, \xi\left(w_{t}\right)=0$ при $t \neq j$. Здесь следует заметить, что $0 \leqslant s \leqslant d_{j} \leqslant d_{i}$ и, таким образом, выражение $e^{d_{i}-s}$ имеет смысл. Необходимо также проверить, что $e^{d_{j}+1}\left(\xi\left(w_{j}\right)\right)=0$. Сложим показатели степеней при $e$ и получим $e^{d_{j}+1}\left(\xi\left(w_{j}\right)\right)=e^{d_{j}+1+d_{i}-s} w_{i}=e^{d_{j}-s}\left(e^{d_{i}+1} w_{i}\right)=0$. Имеем $\alpha([\xi, \varphi])=a_{i} c_{i}^{j, s}-a_{j} c_{i}^{j, d_{j}-d_{i}+s}$. В случае $d_{j}=d_{i}$ мы получаем, что $\left(a_{i}-a_{j}\right) c_{i}^{j, s} \neq 0$. Если же $d_{j}<d_{i}$, то $s>d_{j}-d_{i}+s$. Мы можем выбрать минимальное $s$, при котором $c_{i}^{j, s} \neq 0$. При таком выборе $\alpha([\xi, \varphi])=a_{i} c_{i}^{j, s} \neq 0$.

Пусть теперь $d_{j}>d_{i}$ и $s-$ минимальное, при котором $c_{i}^{j, s} \neq 0$. Положим $\xi\left(w_{j}\right)=e^{d_{j}-s} w_{i}, \xi\left(w_{t}\right)=0$ при $t \neq j$. Как и в предыдущем случае, $0 \leqslant s \leqslant d_{j}$. В частности, $d_{j}-s \geqslant 0, d_{j}+1+d_{j}-s>d_{i}+1$ и, значит, $e^{d_{j}+1}\left(\xi\left(w_{j}\right)\right)=0$. Имеем

$$
\alpha([\varphi, \xi])=a_{j} c_{i}^{j, s}-a_{i} c_{i}^{j, d_{i}-d_{j}+s}=a_{j} c_{i}^{j, s} \neq 0 ;
$$

здесь $c_{i}^{j, d_{i}-d_{j}+s}=0$, так как $d_{i}-d_{j}+s<s$.

СЛЕДСТВИЕ. Индекс алгебри $\mathfrak{z}(e)$ равен $n$.

ДокАзАтЕльство. Стабилизатор $\mathfrak{z}(e)_{\alpha}$ состоит из преобразований, сохраняющих пространства жордановых клеток. В частности, он имеет размерность $n$. 
Следовательно, индекс алгебры $\mathfrak{z}(e)$ не превосходит $n$. С другой стороны, согласно неравенству Винберга, $\operatorname{index} \mathfrak{z}(e) \geqslant n=\operatorname{rk} \mathfrak{g l}(V)$.

Приведем еще одно доказательство неравенства index $\mathfrak{z}(e) \leqslant n$.

Пример 1. Пусть $e \in \mathfrak{g l}_{n}=\mathfrak{g l}(V)-$ нильпотентный элемент и $\mathfrak{h}=\mathfrak{z}(e)-$ его централизатор. Рассмотрим максимальную по размеру жорданову клетку элемента $e$. Будем считать, что она первая. Тогда $V=V_{d_{1}+1} \oplus V_{\text {ост }}, e=e_{1}+e_{2}$, где $V_{d_{1}+1}-$ пространство первой жордановой клетки, а $V_{\text {ост }}-$ пространство всех остальных клеток, причем $e_{1} \in \mathfrak{g l}_{d_{1}+1}, e_{2} \in \mathfrak{g l}_{n-d_{1}-1}$. В качестве инволюции $\tau$ возьмем такое сопряжение диагональной матрицей второго порядка, что $\mathfrak{g l}(V)^{\tau}=\mathfrak{g l}_{d_{1}+1} \oplus \mathfrak{g l}_{n-d_{1}-1}$. Инволюция $\tau$ действует на $\mathfrak{h}=\mathfrak{z}(e)$ и индуцирует разложение $\mathfrak{h}=\mathfrak{h}_{0} \oplus \mathfrak{h}_{1}$, где $\mathfrak{h}_{0}=\mathfrak{z}\left(e_{1}\right) \oplus \mathfrak{z}\left(e_{2}\right)$ (централизаторы рассматриваются в алгебрах $\mathfrak{g l}_{d_{1}+1}$ и $\mathfrak{g l}_{n-d_{1}-1}$ соответственно). Будем считать, что для всех $m<n$ гипотеза об индексе уже доказана, в частности, index $\mathfrak{z}\left(e_{2}\right)=n-d_{1}-1$. Подалгебра $\mathfrak{z}\left(e_{1}\right)$ коммутативна, и ее индекс равен $d_{1}+1$. Согласно лемме 1 , $\operatorname{index} \mathfrak{z}(e) \leqslant \operatorname{index}\left(\mathfrak{z}\left(e_{1}\right) \oplus \mathfrak{z}\left(e_{2}\right)\right)+\min _{\gamma \in \mathfrak{h}_{0}^{*}} \operatorname{dim}(\operatorname{Ker} \hat{\gamma}) \leqslant n+\min _{\gamma \in \mathfrak{z}\left(e_{1}\right)^{*}} \operatorname{dim}(\operatorname{Ker} \hat{\gamma})$. Положим $\gamma\left(\xi_{1}^{1, d_{1}}\right)=1, \gamma\left(\xi_{i}^{j, s}\right)=0$ для всех остальных $\xi_{i}^{j, s}$. Подпространство $\mathfrak{h}_{1}$ порождается векторами $\xi_{i}^{1, s}$ и $\xi_{1}^{i, s}$ при $i \neq 1$. Имеем

$$
\begin{aligned}
\hat{\gamma}\left(\xi_{1}^{i, s}, \xi_{i}^{1, d_{1}-s}\right) & =-1, \\
\hat{\gamma}\left(\xi_{1}^{i, s}, \xi_{i}^{1, b}\right) & =0 \quad \text { при } s+b \neq d_{1}, \\
\hat{\gamma}\left(\xi_{1}^{i, s}, \xi_{1}^{i, b}\right) & =\hat{\gamma}\left(\xi_{i}^{1, s}, \xi_{i}^{1, b}\right)=0 .
\end{aligned}
$$

Форма $\hat{\gamma}$ определяет невырожденное спаривание подпространств $U_{1}^{i}:=\left\langle\xi_{1}^{i, s}\right|$ $\left.0 \leqslant s \leqslant d_{i}\right\rangle$ и $U_{i}^{1}:=\left\langle\xi_{i}^{1, s} \mid d_{1}-d_{i} \leqslant s \leqslant d_{1}\right\rangle$. Следовательно, она невырожденна и $\operatorname{index} \mathfrak{z}(e) \leqslant n$.

\section{§3. Симплектическая алгебра}

Пусть теперь $\mathfrak{g}=\mathfrak{s p}_{2 n}=\mathfrak{s p}(V)$, где $V$ есть $2 n$-мерное линейное пространство над полем $\mathbb{K}$. Как и ранее, $e \in \mathfrak{s p}_{2 n}-$ нильпотентный элемент, а $\mathfrak{z}(e)-$ его централизатор в алгебре $\mathfrak{g}$. Пусть $(,)_{V}-$ невырожденная $\mathfrak{g}$-инвариантная форма на $V$. Можно так выбрать жорданов базис, связанный с элементом $e$, чтобы $(V[i], V[j])_{V}=0$, если $d_{i}$ нечетно и $i \neq j$, а клетки нечетного размера были разбиты на пары $\left(i, i^{\prime}\right)$, где $i \neq i^{\prime}, d_{i}=d_{i^{\prime}}$ и $(V[i], V[j])_{V}=0$, если $j \neq i^{\prime}$.

Пусть $\mathfrak{z}_{\mathfrak{g l}}(e)$ - централизатор элемента $e$ в алгебре $\mathfrak{g l}_{2 n}$. Напомним, что $\mathfrak{z}(e)=\mathfrak{z}_{\mathfrak{g l}}(e)^{\sigma} \oplus \mathfrak{z}_{1}$, где $\sigma$ - инволюция алгебры $\mathfrak{g l}_{2 n}$. Сохраним введенные ранее обозначения для элементов алгебры $\mathfrak{g} \mathfrak{g l}(e)$.

В качестве точки $\alpha \in \mathfrak{z}_{\mathfrak{g l}}(e)^{*}$ возьмем функцию, определенную, как и в случае алгебры $\mathfrak{g l}_{2 n}$ :

$$
\alpha(\varphi)=a_{1} c_{1}^{1, d_{1}}+\cdots+a_{2 n} c_{k}^{k, d_{k}},
$$

где элемент $\varphi$ задается коэффициентами $c_{i}^{j, s}, a_{i}$ - попарно различные ненулевые числа и $a_{i^{\prime}}=-a_{i}$.

ЛЕмма 2. В описанных выше обозначениях $\alpha\left(\mathfrak{z}_{1}\right)=0$.

ДоКАЗАТЕЛЬСтво. Предположим, что найдется $\psi \in \mathfrak{z}_{1}$, такое, что $\alpha(\psi) \neq 0$. Тогда для какого-то $i$ коэффициент $c_{i}^{i, d_{i}}$ элемента $\psi$ не равен нулю. Напомним, 
что $\sigma(\psi)=-\psi$. Элемент $\psi$ «умножает на $-1 » \mathfrak{s p}_{2 n}$-инвариантную кососимметрическую форму $(,)_{V}$, в частности, $\left(\psi\left(w_{i}\right), v\right)_{V}=\left(w_{i}, \psi(v)\right)_{V}$ для любого вектора $v \in V$. Легко видеть, что векторы $\psi\left(w_{i}\right)$ и $w_{i}$ должны быть ортогональны относительно формы $(,)_{V}$. Если $d_{i}$ нечетно, то $\left(w_{i}, e^{d_{i}} w_{i}\right)_{V} \neq 0$. Следовательно, $c_{i}^{i, d_{i}}=0$. Если же $d_{i}$ четно, то

$$
\begin{aligned}
c_{i}^{i, d_{i}}\left(e^{d_{i}} w_{i}, w_{i^{\prime}}\right)_{V} & =\left(\psi\left(w_{i}\right), w_{i^{\prime}}\right)_{V}=\left(w_{i}, \psi\left(w_{i^{\prime}}\right)\right)_{V}=c_{i^{\prime}}^{i^{\prime}, d_{i}}\left(w_{i}, e^{d_{i}} w_{i^{\prime}}\right)_{V} \\
& =(-1)^{d_{i}} c_{i^{\prime}}^{i^{\prime}, d_{i}}\left(e^{d_{i}} w_{i}, w_{i^{\prime}}\right)_{V}=c_{i^{\prime}}^{i^{\prime}, d_{i}}\left(e^{d_{i}} w_{i}, w_{i^{\prime}}\right)_{V} .
\end{aligned}
$$

Следовательно, $c_{i}^{i, d_{i}}=c_{i^{\prime}}^{i^{\prime}, d_{i}}$. Подставив это равенство в формулу, определяющую $\alpha$, мы получим сумму по парам нечетных клеток,

$$
\alpha(\psi)=\sum_{\left(i, i^{\prime}\right)}\left(a_{i}+a_{i^{\prime}}\right) c_{i}^{i, d_{i}},
$$

равную нулю в силу условия $a_{i}=-a_{i^{\prime}}$.

Обозначим через $\tilde{\alpha}$ ограничение функции $\alpha$ на подалгебру $\mathfrak{z}(e)$.

Теорема 2. Размерность стабилизатора $\mathfrak{z}(e)_{\tilde{\alpha}}=\mathfrak{z} \mathfrak{g l}(e)_{\alpha} \cap \mathfrak{s p}_{2 n}$ равна $n$.

ДокАзАтельство. Стабилизатор точки $\alpha$ в алгебре $\mathfrak{z g r}(e)$ состоит из преобразований, сохраняющих пространства жордановых клеток. Согласно предложению $1, \mathfrak{z}(e)_{\tilde{\alpha}}=\mathfrak{z} \mathfrak{g l}(e)_{\alpha} \cap \mathfrak{z}(e)$. Найдем пересечение подалгебры $\mathfrak{z} \mathfrak{g l}(e)_{\alpha} \mathrm{c}$ симплектической подалгеброй. Если $w_{i}$ - образующая клетки четного размера, то элементы $\xi_{i}^{i, s}$ умножают кососимметрическую форму $(,)_{V}$ на $(-1)^{s+1}$, т. е. $\left(\xi_{i}^{i, s}\left(e^{b} w_{i}\right), e^{t} w_{i}\right)_{V}=(-1)^{s}\left(e^{b} w_{i}, \xi_{i}^{i, s}\left(e^{t} w_{i}\right)\right)_{V}$. Рассмотрим пространство, соответствующее паре $\left(i, i^{\prime}\right)$ клеток нечетного размера. Положим $d:=d_{i}=d_{i^{\prime}}$. Напомним, что $\left(w_{i}, e^{d} w_{i^{\prime}}\right)_{V}=(-1)^{s}\left(e^{s} w_{i}, e^{d-s} w_{i^{\prime}}\right)_{V}=-\left(w_{i^{\prime}}, e^{d} w_{i}\right)_{V}$. Поскольку $\left(e^{s} w_{i}, e^{d-s} w_{i^{\prime}}\right)_{V}=(-1)^{s}\left(w_{i}, e^{d} w_{i^{\prime}}\right)_{V}$, элементы $\xi_{i}^{i, s}+(-1)^{s+1} \xi_{i^{\prime}}^{i^{\prime}, s}$ сохраняют форму $(,)_{V}$, а элементы $\xi_{i}^{i, s}+(-1)^{s} \xi_{i^{\prime}}^{i^{\prime}, s}$ умножают ее на -1 . Таким образом, $i$-й клетке четного размера соответствуют $\left(d_{i}+1\right) / 2$ векторов в $\mathfrak{z} \mathfrak{g r}(e)_{\alpha} \cap \mathfrak{s p}_{2 n}$, a паре $\left(i, i^{\prime}\right)$ соответствуют $d_{i}+1$ векторов. Следовательно, во всей алгебре $\mathfrak{s p}_{2 n}$ размерность стабилизатора точки $\alpha$ равняется $n$.

\section{§4. Ортогональная алгебра}

Пусть теперь $\mathfrak{g}=\mathfrak{s o}_{n}=\mathfrak{s o}(V)$. Как и ранее, $e \in \mathfrak{s o}_{n}-$ нильпотентный элемент, а $\mathfrak{z}(e)$ - его централизатор в алгебре $\mathfrak{g}$. Пусть $(,)_{V}$ - невырожденная $\mathfrak{g}$-инвариантная форма на $V$. Можно так выбрать жорданов базис, связанный с элементом $e$, чтобы $(V[i], V[j])_{V}=0$, если $d_{i}$ четно и $i \neq j$, а клетки четного размера были разбиты на пары $\left(i, i^{*}\right)$, где $i \neq i^{*}, d_{i}=d_{i^{*}}$ и $(V[i], V[j])_{V}=0$, если $j \neq i^{*}$. Для клеток нечетного размера положим $i^{*}:=i$.

Как и симплектическая алгебра, $\mathfrak{s o}_{n}$ является симметрической подалгеброй алгебры $\mathfrak{g l}_{n}$. Обозначим задающую ее инволюцию через $\sigma$. Как и ранее, $\sigma(e)=e$ и $\mathfrak{z} \mathfrak{g l}(e)=\mathfrak{z}(e) \oplus \mathfrak{z} 1$. Будем считать, что $\left(w_{i^{*}}, e^{d_{i}} w_{i}\right)_{V}= \pm 1$ и $\left(w_{i}, e^{d_{i}} w_{i}\right)_{V}=1$ при $i=i^{*}$. Алгебра $\mathfrak{z}(e)$ порождается (как линейное пространство) векторами $\xi_{i}^{j, d_{j}-s}+\varepsilon(i, j, s) \xi_{j^{*}}^{i^{*}, d_{i}-s}$, где $\varepsilon(i, j, s)= \pm 1$ в зависимости от $i, j$ и $s$. В свою очередь, пространство $\mathfrak{z}_{1}$ порождается векторами $\xi_{i}^{j, d_{j}-s}-\varepsilon(i, j, s) \xi_{j^{*}}^{i^{*}, d_{i}-s}$. Напомним, что $\left(e^{s} w_{i}, e^{d_{i}-s} w_{i^{*}}\right)_{V} \neq 0$, если $e^{s} w_{i} \neq 0$. 
Приведем некоторые элементарные примеры функций, равных нулю на $\mathfrak{z}_{1}$. Пусть вектор $\varphi \in \mathfrak{z}_{\mathfrak{g l}}(e)$ определяется формулой (1). Положим $\beta_{i}(\varphi)=c_{i}^{i, d_{i}-1}$, $\gamma_{i, j}(\varphi)=c_{i}^{j, d_{j}}$.

Лемма 3. Если $i=i^{*}, j=j^{*}, t \neq t^{*}$, то функции $\beta_{i}, \gamma_{i, j}-\gamma_{j, i} u \gamma_{t, t}+\gamma_{t^{*}, t^{*}}$ тождественно равны нулю на $\mathfrak{z}_{1}$.

ДокАЗАТЕЛЬСтво. Пусть $\psi \in \mathfrak{z}_{1}$ определяется формулой (1). Тогда $\left(\psi\left(w_{i}\right)\right.$, $\left.e w_{i}\right)_{V}=c_{i}^{i, d_{i}-1}\left(e^{d_{1}-1} w_{i}, e w_{i}\right)_{V}$. Так как $\sigma(\psi)=-\psi$, то

$$
\begin{aligned}
\left(\psi\left(w_{i}\right), e w_{i}\right)_{V} & =\left(w_{i}, \psi\left(e w_{i}\right)\right)_{V}=\left(w_{i}, e \psi\left(w_{i}\right)\right)_{V} \\
& =-\left(e w_{i}, \psi\left(w_{i}\right)\right)_{V}=-c_{i}^{i, d_{i}-1}\left(e w_{i}, e^{d_{1}-1} w_{i}\right)_{V} .
\end{aligned}
$$

В силу неравенства $\left(e w_{i}, e^{d_{i}-1} w_{i}\right)_{V} \neq 0$ и симметричности формы $(,)_{V}$ получаем $\beta_{i}(\psi)=c_{i}^{i, d_{i}-1}=0$.

Аналогично,

$$
\begin{aligned}
c_{i}^{j, d_{j}}\left(e^{d_{j}} w_{j}, w_{j}\right)_{V} & =\left(\psi\left(w_{i}\right), w_{j}\right)_{V}=\left(w_{i}, \psi\left(w_{j}\right)\right)_{V}=c_{j}^{i, d_{i}}\left(w_{i}, e^{d_{i}} w_{i}\right)_{V}, \\
c_{t}^{d_{t}, t}\left(e^{d_{t}} w_{t}, w_{t *}\right)_{V} & =\left(\psi\left(w_{t}\right), w_{t^{*}}\right)_{V}=\left(w_{t}, \psi\left(w_{t^{*}}\right)\right)_{V}=c_{t^{*}}^{t^{*}, d_{t}}\left(w_{t}, e^{d_{t}} w_{t^{*}}\right)_{V} .
\end{aligned}
$$

Напомним, что, согласно нашему выбору, $\left(e^{d_{j}} w_{j}, w_{j}\right)_{V}=\left(w_{i}, e^{d_{i}} w_{i}\right)_{V}=1,\left(e^{d_{t}} w_{t}\right.$, $\left.w_{t *}\right)_{V}=-\left(w_{t}, e^{d_{t}} w_{t^{*}}\right)_{V}$. Следовательно, $c_{i}^{j, d_{j}}=c_{j}^{i, d_{i}}, c_{t}^{t, d_{t}}=-c_{t^{*}}^{t^{*}, d_{t}}$.

Докажем неравенство index $\mathfrak{z}(e) \leqslant \operatorname{rkso} \mathfrak{s}_{n}$, используя полную индукцию по $n$. В следующих двух случаях шаг индукции невозможен, поэтому разберем их отдельно.

Первый случай. Если $e \in \mathfrak{s o}_{2 m+1}-$ регулярный нильпотентный элемент, то $\mathfrak{z}(e)$ - коммутативная алгебра размерности $m$.

Bторой случай. Пусть $e \in \mathfrak{s o}_{4 d}-$ нильпотентный элемент, имеющий две жордановы клетки размера $2 d$. Положим $\alpha(\varphi)=c_{1}^{1,2 d-2}-c_{2}^{2,2 d-2}$, где элемент $\varphi$ определяется по формуле (1). Нетрудно проверить, что $\mathfrak{z}(e)_{\alpha}$ имеет следующий базис: $\xi_{1}^{1, s}+(-1)^{s+1} \xi_{2}^{2, s}$ при $0 \leqslant s \leqslant 2 d-1$, и $\operatorname{dim} \mathfrak{z}(e)_{\alpha}=2 d$.

Упорядочим клетки по убыванию размера: $d_{1} \geqslant \cdots \geqslant d_{k}$. Здесь, как и в случае полной линейной алгебры, $d_{i}+1-$ размерность пространства $i$-й жордановой клетки. Заметим, что числа $n$ и $k$ всегда имеют одинаковую четность. Будем считать, что $k>1$ и что в случае $k=2$ обе клетки имеют нечетный размер. Тогда имеет место одна из следующих возможностей:

1) для некоторого четного числа $2 p<k$ ограничение формы $(,)_{V}$ на пространство, соответствующее первым $2 p$ клеткам, невырожденно;

2 ) число $d_{i}$ четно при $i=1, k$ и нечетно для всех остальных клеток;

3 ) число $d_{i}$ четно только при $i=1$.

Каждая из этих трех возможностей разобрана отдельно. В первых двух случаях делается шаг индукции, а в третьем предъявлена точка $\alpha \in \mathfrak{z}(e)^{*}$, для которой $\operatorname{dim} \mathfrak{z}(e)_{\alpha} \leqslant \operatorname{rk} \mathfrak{s o}_{n}$.

1) Пусть пространство $V_{2 m}$, соответствующее первым $2 p$ клеткам, имеет размерность $2 m$ и ограничение формы $(,)_{V}$ на него невырожденно. Тогда $V=$ $V_{2 m} \oplus V_{\text {ост }}, e=e_{1}+e_{2}$, где $e_{1} \in \mathfrak{s o}_{2 m}, e_{2} \in \mathfrak{s o}_{n-2 m}$. Рассмотрим инволюцию $\tau$ алгебры $\mathfrak{g l}_{n}$, соответствующую данной прямой сумме, т. е. $\mathfrak{g l} \mathfrak{l}_{n}^{\tau}=\mathfrak{g l}\left(V_{2 m}\right) \oplus \mathfrak{g l}\left(V_{\text {ост }}\right)$. Пусть $\mathfrak{h}=\mathfrak{z}(e), \mathfrak{h}_{0}=\mathfrak{z}(e)^{\tau}$. Тогда $\mathfrak{h}_{0}=\mathfrak{z}\left(e_{1}\right) \oplus \mathfrak{z}\left(e_{2}\right)$, где централизаторы рассматриваются в алгебрах $\mathfrak{s o}_{2 m}$ и $\mathfrak{s o}_{n-2 m}$ соответственно. По предположению 
индукции $\operatorname{index} \mathfrak{z}\left(e_{1}\right)=m, \operatorname{index} \mathfrak{z}\left(e_{2}\right)=[n / 2]-m$. Следовательно, index $\mathfrak{z}(e) \leqslant$ $[n / 2]+\min _{\gamma \in \mathfrak{h}_{0}^{*}} \operatorname{dim}(\operatorname{Ker} \hat{\gamma})$. Осталось предъявить точку $\gamma \in \mathfrak{h}_{0}^{*}$, для которой форма $\hat{\gamma}$ невырожденна. Напомним, что инволюции $\sigma$ и $\tau$ коммутируют, сохраняют $е$ и задают разложение алгебры $\mathfrak{z} \mathfrak{g l}(e)=\left(\mathfrak{z}\left(e_{1}\right) \oplus \mathfrak{z}\left(e_{2}\right) \oplus \mathfrak{h}_{1}\right) \oplus \mathfrak{z} 1$. Если $\gamma\left(\mathfrak{z}_{1}\right)=\gamma\left(\mathfrak{h}_{1}\right)=0$, то Ker $\hat{\gamma}=\mathfrak{h}_{1} \cap \mathfrak{z}_{\mathfrak{g l}}(e)_{\gamma}$.

Разобьем клетки нечетного размера на пары $\left(i, i^{\prime}\right)$ (здесь предполагается, что $\left.i, i^{\prime} \leqslant 2 p\right)$. Определим точку $\gamma$ формулой

$$
\gamma(\varphi)=\sum_{\left(i, i^{\prime}\right), i, i^{\prime} \leqslant 2 p}\left(c_{i}^{i^{\prime}, d_{i^{\prime}}}-c_{i^{\prime}}^{i, d_{i}}\right)+\sum_{j \leqslant 2 p,\left(d_{j}+1\right)-\text { четное }} c_{j}^{j, d_{j}},
$$

где элемент $\varphi \in \mathfrak{z} \mathfrak{g r}(e)$ задается коэффициентами $c_{i}^{j, s}$. Первое слагаемое является суммой функций $\gamma_{i, i^{\prime}}-\gamma_{i^{\prime}, i}$ по парам клеток нечетного размера, второе - суммой функций $\gamma_{j, j}+\gamma_{j^{*}, j^{*}}$ по парам клеток четного размера. Согласно лемме 3 , оба слагаемых тождественно равны нулю на $\mathfrak{z}$. Кроме того, по определению $\gamma\left(\mathfrak{h}_{1}\right)=0$.

Для клеток четного размера положим $j^{\prime}:=j$. Предположим, что элемент $\psi \in \mathfrak{h}_{1}$, определенный по формуле $(1)$, лежит в ядре формы $\hat{\gamma}$, т. е. $\gamma\left(\left[\psi, \mathfrak{h}_{1}\right]\right)=0$. Тогда $\gamma([\psi, \mathfrak{h}])=\gamma([\psi, \mathfrak{z} \mathfrak{g l}(e)])=0$. Поскольку $\psi \in \mathfrak{s o}_{n}$ и $\psi \neq 0$, можно считать, что для некоторых $j>2 p \geqslant i$ коэффициент $c_{i}^{j, s}$ не равен нулю. Имеем

$$
\gamma\left(\left[\psi, \eta_{i}^{j^{\prime}, d_{j^{\prime}}}\right]\right)= \pm c_{i}^{j, s} \neq 0 .
$$

Доказано, что форма $\hat{\gamma}$ невырожденна и $\operatorname{index} \mathfrak{z}(e) \leqslant[n / 2]$.

2) Рассмотрим разложение $V=V_{\text {ост }} \oplus V_{d_{k}+1}$, где последнее слагаемое - это пространство самой маленькой клетки, а первое соответствует всем остальным клеткам. Как и ранее, $e=e_{1}+e_{2}$, где $e_{2} \in \mathfrak{s o}\left(V_{d_{k}+1}\right)$. Инволюцию $\tau$, алгебры $\mathfrak{z}\left(e_{1}\right), \mathfrak{z}\left(e_{2}\right), \mathfrak{h}_{0}$ и подпространство $\mathfrak{h}_{1}$ определим так же, как и в случае 1$)$. По предположению индукции $\operatorname{index} \mathfrak{z}\left(e_{1}\right)=\left[\left(n-d_{k}-1\right) / 2\right], \operatorname{index} \mathfrak{z}\left(e_{2}\right)=d_{k} / 2$. Следовательно, index $\mathfrak{h}_{0}=n / 2-1$. Согласно лемме $1, \operatorname{index} \mathfrak{z}(e) \leqslant \operatorname{index} \mathfrak{h}_{0}+$ $\min _{\gamma \in \mathfrak{h}_{0}^{*}} \operatorname{dim}(\operatorname{Ker} \hat{\gamma})$. В качестве $\gamma$ возьмем следующую функцию:

$$
\gamma(\varphi)=c_{1}^{1, d_{1}-1}+\sum_{i=2}^{k-1} c_{i}^{i, d_{i}}
$$

где элемент $\varphi$ определен по формуле (1). Первое слагаемое - это $\beta_{1}$, второе является суммой функций $\gamma_{j, j}+\gamma_{j^{*}, j^{*}}$ по парам клеток четного размера. Согласно лемме $3, \gamma\left(\mathfrak{z}_{1}\right)=0$. Пусть $\psi \in \mathfrak{h}_{1}$ определяется коэффициентами $c_{i}^{j, s}$. Имеем

$$
\begin{aligned}
& \gamma\left(\left[\psi, \xi_{1}^{k, b}\right]\right)=c_{k}^{1, d_{1}-1-b}, \\
& \gamma\left(\left[\psi, \xi_{i}^{k, b}\right]\right)=c_{k}^{i, d_{i}-b} \quad \text { при } 1<i<k .
\end{aligned}
$$

Легко видеть, что ядро формы $\hat{\gamma}$ одномерно и порождено вектором $\xi_{k}^{1, d_{1}}-\xi_{1}^{k, d_{k}}$. Следовательно, index $\mathfrak{z}(e) \leqslant[n / 2]-1+1=[n / 2]$.

3) Пусть теперь $n$ и $k$ нечетны и элемент $e$ имеет всего одну клетку нечетного размера, причем максимального. Пусть $k=2 m+1$. Занумеруем клетки целыми числами от $-m$ до $m$ так, чтобы единственная клетка нечетного размера имела номер $0 ; i^{*}=-i$ для всех $i \neq 0$; пространства $V[i]$ и $V[j]$ были ортогональны при $i+j \neq 0 ; i\left(w_{i}, e^{d_{i}} w_{-i}\right)_{V}=|i|$ при $i \neq 0$ и $\left(w_{0}, e^{d_{0}} w_{0}\right)_{V}=1$; размеры клеток 
возрастали для номеров от $-m$ до 0 и убывали для номеров от 0 до $m$, т. е. если $|i| \leqslant|j|$, то $d_{i} \geqslant d_{j}$. Заметим, что $d_{i}=d_{-i}$. Такая нумерация клеток показана на рис. 1.

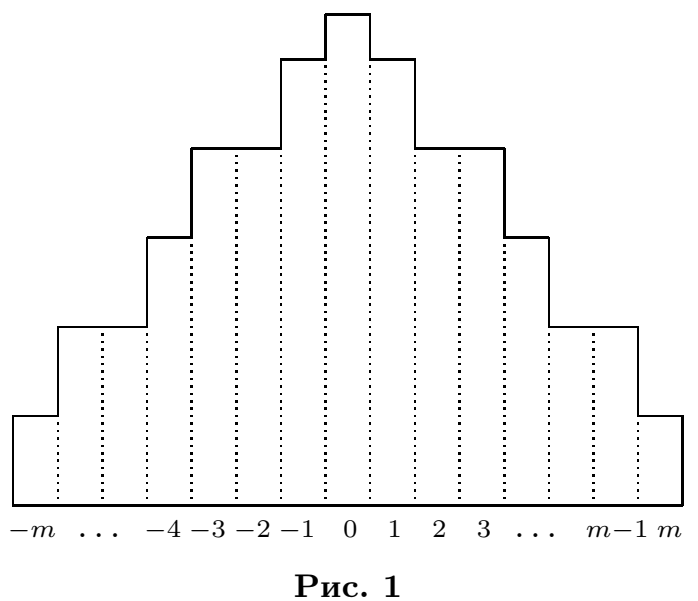

Пусть элемент $\varphi \in \mathfrak{g} \mathfrak{g l}(e)$ задается формулой (1). Рассмотрим следующую точку $\alpha \in \mathfrak{z}_{\mathfrak{g l}}(e)^{*}$ :

$$
\alpha(\varphi)=\sum_{i=-m+1}^{m} c_{i-1}^{i, d_{i}} .
$$

Непосредственным вычислением можно убедиться, что $c_{i-1}^{i, d_{i}}(\psi)=-c_{-i}^{1-i, d_{1-i}}(\psi)$ для каждого $\psi \in \mathfrak{z}_{1}$ и, следовательно, $\alpha\left(\mathfrak{z}_{1}\right)=0$. Пусть $\tilde{\alpha} \in \mathfrak{z}(e)^{*}-$ ограничение функции $\alpha$. Опишем стабилизатор $\mathfrak{z}(e)_{\tilde{\alpha}}=\mathfrak{z} \mathfrak{g r}(e)_{\alpha} \cap \mathfrak{z}(e)$. Отметим, что $\alpha\left(\left[\xi_{i}^{j, s}, \varphi\right]\right)=c_{j-1}^{i, d_{j}-s}(\varphi)-c_{j}^{i+1, d_{i+1}-s}(\varphi)$.

Лемма 4. Пусть $\varphi \in \mathfrak{z}(e) u \operatorname{ad}^{*}(\varphi) \alpha=0$. Тогда $c_{i}^{j, s}=c_{i}^{j, s}(\varphi)=0$ nрu $i<j$.

ДокАзАтЕЛЬСтво. Предположим, что утверждение неверно, и рассмотрим максимальное $i$, для которого существуют такие $j>i$ и $s$, что $c_{i}^{j, s} \neq 0$. Так как $\varphi$ сохраняет форму $(,)_{V}$, то $c_{-j}^{-i, d_{i}-d_{j}+s}= \pm c_{i}^{j, s} \neq 0$. Следовательно, $-j \leqslant i$ $<j, j>0,|i| \leqslant j$ и $d_{i} \geqslant d_{j}$. Более того, $-j<(i+1) \leqslant j$ и $d_{i+1} \geqslant d_{j}$. Легко видеть, что $d_{i+1}-s \geqslant d_{j}-s \geqslant 0$ и существует элемент $\xi_{j}^{i+1, d_{i+1}-s} \in \mathfrak{z} \mathfrak{g l}(e)$. Получаем

$$
0=\alpha\left(\left[\xi_{j}^{i+1, d_{i+1}-s}, \varphi\right]\right)=c_{i}^{j, s}-c_{i+1}^{j+1, \delta}=c_{i}^{j, s} ;
$$

не будем уточнять, чему равняется $\delta$, так как все коэффициенты $c_{i+1}^{j+1, b}$ равны нулю, поскольку $j+1>i+1>i$. Мы получили противоречие. Тем самым утверждение леммы доказано.

Будем говорить, что элемент $\varphi \in \mathfrak{z} \mathfrak{g l}(e)$ имеет шаг $l$, если $c_{i}^{j, s}(\varphi) \neq 0$ только при $j=i+l$. Вектор $\varphi \in \mathfrak{z g l}(e)$ можно представить в виде суммы $\varphi=\varphi_{-2 m}+$ $\varphi_{-2 m+1}+\cdots+\varphi_{2 m-1}+\varphi_{2 m}$, где $\varphi_{l}$ имеет шаг $l$. Так как $(-i)-(-j)=j-i$, шаг корректно определен на алгебре $\mathfrak{z}(e)$. Из определения точки $\alpha$ следует, что $\alpha\left(\varphi_{l}, \varphi_{t}\right) \neq 0$ только при $l+t=1$. Стабилизатор $\mathfrak{z}(e)_{\alpha}$ является прямой суммой своих подпространств $\Phi_{l}$, каждое из которых состоит из преобразований шага $l$. 
Как было показано, $\Phi_{l}=\varnothing$ при $l>0$. Осталось описать преобразования с неположительными шагами.

Пример 2. Покажем, что $\operatorname{dim} \Phi_{0} \leqslant d_{0} / 2$. Пусть $\varphi \in \Phi_{0}, \varphi \neq 0$ и $\varphi\left(w_{0}\right)=0$. Рассмотрим минимальное по модулю число $i$, для которого $\varphi\left(w_{i}\right) \neq 0$. Так как $\varphi \in \mathfrak{s o}_{n}$, то также $\varphi\left(w_{-i}\right) \neq 0$. Будем считать, что $i>0$ и коэффициент $c_{i}^{i, s}$ элемента $\varphi$ отличен от нуля. Тогда $|i-1|<i, d_{i-1} \geqslant d_{i}$, существует элемент $\xi:=\xi_{i-1}^{i, d_{i}-s}$ и $0=\alpha([\varphi, \xi])=c_{i}^{i, s}-c_{i-1}^{i-1, s}=c_{i}^{i, s}$. Следовательно, если $\varphi\left(w_{0}\right)=0$, то и $\varphi=0$. Получаем, что векторы пространства $\Phi_{0}$ полностью определяются своими значениями на $w_{0}$. В свою очередь, $\varphi\left(w_{0}\right)=c_{1} e w_{0}+c_{3} e^{3} w_{0}+\cdots+$ $c_{d_{0}-1} e^{d_{0}-1} w_{0}$.

ЛЕмма 5. Если $q=2 l$ или $q=2 l-1$, где $0<l \leqslant m$, mo $\operatorname{dim} \Phi_{-q} \leqslant\left(d_{l}+1\right) / 2$.

ДокАЗАтЕльство. Аналогично разобранному примеру покажем, что если $\varphi \in \Phi_{-q}$ и $\varphi\left(w_{l}\right)=0$, то и $\varphi=0$. Если $\varphi\left(w_{i}\right) \neq 0$, то и $\varphi\left(w_{q-i}\right) \neq 0$, так как $\varphi \in$ $\mathfrak{s o}_{n}$. Пусть $\varphi\left(w_{j}\right) \neq 0$ для некоторого номера $j$. Если $j<l$, то $j-q \geqslant l$, однако $\varphi\left(w_{l}\right)=0$; следовательно, $j>l$. Найдем минимальное $j>l$, для которого $\varphi\left(w_{j}\right) \neq 0$. Пусть $c_{j}^{j-q, s}=c_{j}^{j-q, s}(\varphi) \neq 0$. Имеем $-j<-l \leqslant j-q-1<j$, $d_{j} \leqslant d_{j-q-1}$ и $d_{j-q}-s \geqslant 0$. Следовательно, существует $\xi:=\xi_{j-q-1}^{j, d_{j-q}-s} \in \mathfrak{z} \mathfrak{g l}(e)$. Как и в примере $2,0=\alpha([\varphi, \xi])=c_{j}^{j-q, s}-c_{j-1}^{j-q-1, \delta}=c_{j}^{j-q, s}$ (не будем уточнять, чему равняется $\delta$, ведь $\varphi\left(w_{j-1}\right)=0$, поскольку $\left.l \leqslant j-1<j\right)$.

Осталось выяснить какие значения принимает $\varphi\left(w_{l}\right)$. Если $q=2 l$, то $\varphi\left(w_{l}\right)=$ $c_{0} w_{-l}+c_{2} e^{2} w_{-l}+\ldots+c_{d_{l}} e^{d_{l}} w_{-l}$. В случае $q=2 l-1$ получаем следующее уравнение на коэффициенты элемента $\varphi: 0=\alpha\left(\left[\varphi, \xi_{-l}^{l, b}\right]\right)=c_{l}^{-l+1, d_{l-1}-b}-c_{l-1}^{-l, d_{l}-b}$, т. е. $c_{l}^{-l+1, d_{l-1}-b}=c_{l-1}^{-l, d_{l}-b}$. Такое возможно только при нечетном $b$.

Теорема 3. Пусть $е \in \mathfrak{s o}_{n}-$ нильпотентный элемент. Тогда index $\mathfrak{z}(e)=$ $\operatorname{rk} \mathfrak{s o}_{n}=[n / 2]$.

ДокАзАТЕЛьСтво. Если имеет место возможность 3), то, как было показано, $\mathfrak{z}(e)_{\tilde{\alpha}}=\bigoplus_{q=0}^{2 m} \Phi_{-q}$. Кроме того, $\operatorname{dim} \Phi_{q}$ не превосходит половины размера жордановой клетки с номером $[(q+1) / 2]$. Таким образом, $\operatorname{dim} \mathfrak{z}(e)_{\tilde{\alpha}} \leqslant$ $\left(d_{0}+1\right) / 2+\sum_{l=1}^{m} d_{l}=[n / 2]$. С другой стороны, согласно неравенству Винберга, $\operatorname{index} \mathfrak{z}(e) \geqslant[n / 2]$.

В случаях 1) и 2) неравенство index $\mathfrak{z}(e) \leqslant r k \mathfrak{s o}_{n}$ было доказано по индукции.

Если же ни одна из этих трех возможностей не имеет места, то либо $k=1$ и $e$ - регулярный нильпотент, либо $k=2$ и обе жордановы клетки элемента $e$ имеют четный размер. Эти два случая были разобраны отдельно.

\section{§5. Точки общего положения}

В данном параграфе предполагается, что поле $\mathbb{K}$ алгебраически замкнуто и его характеристика равна нулю. Пусть задано линейное действие алгебры Ли $\mathfrak{g}$ на векторном пространстве $V$.

ОПРЕДЕЛЕНИЕ. Вектор $x \in V$ (подалгебра $\mathfrak{g}_{x}$ ) называется точкой общего положения (стабилизатором общего положения), если для точек $y \in U \subset V$ некоторого открытого по Зарисскому подмножества $U$ алгебры $\mathfrak{g}_{y}$ и $\mathfrak{g}_{x}$ сопряжены в $\mathfrak{g}$. 
Хорошо известно, что точка общего положения существует для любого линейного действия редуктивной алгебры Ли.

В работе [4, §1] Элашвили доказал, что подалгебра $\mathfrak{g}_{x}$ является стабилизатором общего положения тогда и только тогда, когда $V=V^{\mathfrak{g}_{x}}+\mathfrak{g} x$, где $V^{\mathfrak{g}_{x}}$ - множество неподвижных относительно действия подалгебры $\mathfrak{g}_{x}$ векторов пространства $V$. Товель и Ю заметили, что в случае коприсоединенного представления $\mathfrak{g} x=\left(\mathfrak{g} / \mathfrak{g}_{x}\right)^{*}=\operatorname{Ann}\left(\mathfrak{g}_{x}\right),\left(\mathfrak{g}^{*}\right)^{\mathfrak{g}_{x}}=\operatorname{Ann}\left(\left[\mathfrak{g}_{x}, \mathfrak{g}\right]\right)$. Это позволило им получить простой и удобный критерий.

TeOpema 4 [13, Corollaire 1.8]. Пусть $\mathfrak{g}-$ алгебра Ли $u x \in \mathfrak{g}^{*}$. Подалгебра $\mathfrak{g}_{x}$ является стабилизатором общего положения коприсоединенного представления алгебры $\mathfrak{g}$ тогда и только тогда, когда $\left[\mathfrak{g}_{x}, \mathfrak{g}\right] \cap \mathfrak{g}_{x}=\{0\}$.

$\mathrm{K}$ сожалению, авторы работы [13] не были знакомы с упомянутым выше результатом Элашвили и доказали его заново.

Пусть $e \in \mathfrak{g l}_{n}-$ нильпотентный элемент и $\mathfrak{z}(e)$ - его централизатор. Пусть $\mathfrak{h}=\mathfrak{z}(e)_{\alpha}$, где точка $\alpha \in \mathfrak{z}(e)^{*}$ определена так же, как и в $\S 2$.

ПреДЛОЖЕНИЕ 2. Имеет место $\mathfrak{h}$-инвариантное разложение $\mathfrak{z}(e)=\mathfrak{h} \oplus \mathfrak{m}$, где пространство $\mathfrak{m}$ порождается векторами $\xi_{i}^{j, s}$ при $i \neq j$.

ДокАзАТЕЛЬСтво. Напомним, что алгебра $\mathfrak{h}$ порождается векторами $\xi_{i}^{i, s}$. Включение $[\mathfrak{h}, \mathfrak{m}] \subset \mathfrak{m}$ непосредственно следует из равенств

$$
\left[\xi_{i}^{i, s}, \xi_{j}^{t, b}\right]= \begin{cases}-\xi_{i}^{t, s+b} & \text { при } i=j, i \neq t, \\ \xi_{j}^{i, s+b} & \text { при } i=t, i \neq j, \\ 0 & \text { в остальных случаях. }\end{cases}
$$

Аналогичное разложение имеет место и в случае симплектической алгебры. Пусть $e \in \mathfrak{s} \mathfrak{p}(V) \subset \mathfrak{g l}(V)$. Сохраним обозначения из $\S 3$. Пусть $\mathfrak{z}_{\mathfrak{g l}}(e)=\mathfrak{h} \oplus \mathfrak{m}$. Легко видеть, что это разложение $\sigma$-инвариантно и $\mathfrak{z}_{\mathfrak{s p}}(e)=\mathfrak{h}^{\sigma} \oplus \mathfrak{m}^{\sigma}$, где $\mathfrak{h}^{\sigma}=$ $\mathfrak{z}_{\mathfrak{s p}}(e)_{\tilde{\alpha}}$.

Теорема 5. Построенные в случаях полной линейной и симплектической алгебр подалгебры $\mathfrak{z}_{\mathfrak{g l}}(e)_{\alpha} \quad$ u $\mathfrak{z}_{\mathfrak{s p}}(e)_{\tilde{\alpha}}$ являются стабилизаторами общего положения коприсоединенных действий алгебр $\mathfrak{z}_{\mathfrak{g l}}(e) u \mathfrak{z}_{\mathfrak{s p}}(e)$.

ДокАЗАТЕЛЬСТво. Проверим, что выполнено условие теоремы 4. Поскольку $\left[\mathfrak{h}, \mathfrak{z}_{\mathfrak{g l}}(e)\right]=[\mathfrak{h}, \mathfrak{m}] \subset \mathfrak{m}$, имеем $\left[\mathfrak{h}, \mathfrak{z}_{\mathfrak{g l}}(e)\right] \cap \mathfrak{h}=0$. Аналогично, $\left[\mathfrak{h}^{\sigma}, \mathfrak{z}_{\mathfrak{s} p}(e)\right] \subset \mathfrak{m}^{\sigma}$.

В ортогональном случае стабилизатор общего положения коприсоединенного действия алгебры $\mathfrak{z}(e)$ может и не существовать.

ПримеР 3. Пусть $e \in \mathfrak{s o}_{8}-$ субрегулярный нильпотентный элемент. Тогда он имеет две жордановы клетки размера 3 и 5 . Нормируем образующие жордановых клеток так, что $\left(w_{1}, e^{2} w_{1}\right)_{V}=\left(w_{2}, e^{4} w_{2}\right)_{V}=1$. Алгебра $\mathfrak{z}(e)$ имеет размерность 6 и трехмерный центр, порожденный векторами $e, e^{3}=\xi_{2}^{2,3}$ и $\varphi_{3}=\xi_{1}^{2,4}-\xi_{2}^{1,2}$. Поскольку $\operatorname{index} \mathfrak{z}(e)=4$, в точках некоторого открытого подмножества $\alpha \in U \subset \mathfrak{z}(e)^{*}$ выполнено равенство $\operatorname{dim} \mathfrak{z}(e)_{\alpha}=4$.

Предположим, что существует стабилизатор общего положения коприсоединенного действия алгебры $\mathfrak{z}(e)$, и обозначим его через $\mathfrak{f}$. Ясно, что $\mathfrak{f}$ содержит центр алгебры $\mathfrak{z}(e)$. Рассмотрим элемент $\varphi_{2}=\xi_{1}^{2,3}+\xi_{2}^{1,1} \in \mathfrak{z}(e)$. Легко видеть, что подпространство $\left[\varphi_{2}, \mathfrak{z}(e)\right]$ является линейной оболочкой векторов $e^{3}$ и $\varphi_{3}$. 
В частности, оно содержится в центре алгебры $\mathfrak{z}(e)$ и, таким образом, в $\mathfrak{f}$. Следовательно, $\left[\varphi_{2}, \mathfrak{f}\right] \subset \mathfrak{f}$ и, согласно теореме $4, \mathfrak{f} \subset \mathfrak{z}(e)_{\varphi_{2}}$. Поскольку $\operatorname{dim} \mathfrak{z}(e)_{\varphi_{2}}=4$, имеет место равенство $\mathfrak{f}=\mathfrak{z}(e)_{\varphi_{2}}$. Однако $\mathfrak{z}(e)_{\varphi_{2}}=\left\langle e, e^{3}, \varphi_{3}, \varphi_{2}\right\rangle_{\mathbb{K}}$ является нормальной, но не центральной подалгеброй в $\mathfrak{z}(e)$.

Рассмотрим вложение алгебры $\mathfrak{s o}_{8}$ в $\mathfrak{s o}_{9}$ в качестве стабилизатора первого базисного вектора в $\mathbb{K}^{9}$. Аналогичным рассуждением можно показать, что коприсоединенное действие алгебры $\mathfrak{z}_{\mathfrak{s o}}(e)$ не имеет стабилизатора общего положения.

\section{§6. Коммутаторные многообразия}

Пусть $\mathfrak{g}$ - алгебра Ли над алгебраически замкнутым полем $\mathbb{K}$ нулевой характеристики. Замкнутое подмножество $Y=\{(x, y) \mid x, y \in \mathfrak{g},[x, y]=0\} \subset \mathfrak{g} \times \mathfrak{g}$ называется коммутаторным многообразием алгебры $\mathfrak{g}$. Вопрос о неприводимости многообразия $Y$ вызывает большой интерес. В случае редуктивной алгебры $\mathfrak{g}$ это многообразие $Y$ неприводимо и совпадает с замыканием $\overline{G(\mathfrak{a}, \mathfrak{a})}$ разнесения $G(\mathfrak{a}, \mathfrak{a})$, где $\mathfrak{a} \subset \mathfrak{g}$ - картановская подалгебра, $G$ - связная группа Ли и Lie $G=\mathfrak{g}$.

Пусть $e \in \mathfrak{g l}_{n}$ - нильпотентный элемент и $\mathfrak{z}(e)$ - его централизатор. Сохраним обозначения $\S 2$. Пусть $\mathfrak{h}=\mathfrak{z}(e)_{\alpha}$. Рассмотрим подалгебру $\mathfrak{t} \subset \mathfrak{z}(e)$, порожденную векторами $\xi_{i}^{i, 0}$. Легко видеть, что $\mathfrak{t} \subset \mathfrak{h}$. Более того, так как $\left[\xi_{i}^{j, s}, t_{i} \xi_{i}^{i, 0}+t_{j} \xi_{j}^{j, 0}\right]=\left(t_{i}-t_{j}\right) \xi_{i}^{j, s}$, алгебра $\mathfrak{h}$ совпадет с нормализатором (централизатором) подалгебры $\mathfrak{t}$ в $\mathfrak{z}(e)$. Следовательно, алгебра $\mathfrak{z}(e)_{\alpha}$ совпадает со своим нормализатором в алгебре $\mathfrak{z}(e)$.

Пусть $Z(e)$ - связная компонента централизатора элемента $e$ в $\mathrm{GL}_{n}$. Тогда $Y_{0}=\overline{Z(e)(\mathfrak{h}, \mathfrak{h})} \subset Y-$ неприводимая компонента максимальной размерности. Следовательно, $Y$ неприводимо тогда и только тогда, когда $Y_{0}=Y$. Известно, что если нильпотент $e$ имеет не более двух жордановых клеток, то $Y$ неприводимо [7]. В общем случае это неверно, так как привело бы к неприводимости многообразия троек коммутирующих матриц.

ПримеР 4. Предположим, что $Y_{0}=Y$ для всех нильпотентных элементов $e \in \mathfrak{g l}_{m}$ при $m \leqslant n$. Рассмотрим множество троек коммутирующих матриц

$$
C_{3}=\left\{(A, B, C) \mid A, B, C \in \mathfrak{g l}_{n},[A, B]=[A, C]=[B, C]=0\right\} .
$$

Пусть $\mathfrak{a} \subset \mathfrak{g l}_{n}$ - подалгебра диагональных матриц. Легко видеть, что $\overline{\mathrm{GL}_{n}(\mathfrak{a}, \mathfrak{a}, \mathfrak{a})}$ - неприводимая компонента многообразия $C_{3}$. Докажем по индукции, что она совпадает с $C_{3}$. При $n=1$ доказывать нечего. Пусть $n>1$. Покажем, что каждая тройка $(A, B, C)$ коммутирующих матриц лежит в замыкании $\overline{\mathrm{GL}_{n}(\mathfrak{a}, \mathfrak{a}, \mathfrak{a})}$. Без ограничения общности можно считать, что $A, B, C \in \mathfrak{s l}_{n}$. Пусть $A=A_{s}+$ $A_{n}$ - разложение Жордана матрицы $A$. Если $A_{s} \neq 0$, то рассмотрим его централизатор $\mathfrak{z}\left(A_{s}\right)$ в алгебре $\mathfrak{g l}_{n}$. Легко видеть, что $A, B, C \in \mathfrak{z}\left(A_{s}\right)$ и $\mathfrak{z}\left(A_{s}\right)$ является суммой нескольких алгебр $\mathfrak{g l}_{n_{i}}$ меньшей размерности. Можно считать, что $\mathfrak{a} \subset \mathfrak{z}\left(A_{s}\right)$. Тогда по предположению индукции

$$
(A, B, C) \in \overline{Z\left(A_{s}\right)(\mathfrak{a}, \mathfrak{a}, \mathfrak{a})} \subset \overline{\mathrm{GL}_{n}(\mathfrak{a}, \mathfrak{a}, \mathfrak{a})} .
$$

Пусть теперь все три элемента $A, B, C$ нильпотенты и хотя бы один из них, например $A$, не является регулярным. Рассмотрим централизатор $\mathfrak{z}(A) \subset \mathfrak{g l}_{n}$. Мы предположили, что $Y_{0}=Y$, т. е. пара $(B, C)$ лежит в замыкании разнесения 
$Z(A)(\mathfrak{h}, \mathfrak{h})$. Достаточно показать, что $(A, \mathfrak{h}, \mathfrak{h}) \subset \overline{\operatorname{GL}_{n}(\mathfrak{a}, \mathfrak{a}, \mathfrak{a})}$. Пусть $x \in \mathfrak{t} \subset \mathfrak{h}-$ полупростой элемент, не лежащий в центре алгебры $\mathfrak{g l}_{n}$. Тогда $A \in\left(\mathfrak{g l}_{n}\right)_{x}$ и $\mathfrak{h} \subset\left(\mathfrak{g l}_{n}\right)_{x}$. Мы снова можем сделать шаг индукции, перейдя к подалгебре $\left(\mathfrak{g l}_{n}\right)_{x}$.

Если же все три элемента $A, B, C$-регулярные нильпотенты, то некоторая их нетривиальная линейная комбинация $A^{\prime}$ является нерегулярным нильпотентом. В частности, тройка $(A, B, C)$ эквивалентна относительно действия $\mathrm{GL}_{n}$ некоторой тройке $\left(A^{\prime}, B^{\prime}, C^{\prime}\right)$ коммутирующих матриц.

Известно, что при $n>31$ многообразие $C_{3}$ приводимо, см. [6]. Следовательно, заведомо для некоторых нильпотентов многообразие $Y$ также приводимо. Было бы интересно найти минимальные (в некотором смысле) нильпотенты, для которых $Y$ приводимо и/или описать какие-либо классы нильпотентов, для которых $Y$ неприводимо.

\section{ЛитЕРАТУРА}

1. Болсинов A. B. Критерий полноты семейства функций в инволюции, построенного методом сдвига аргумента. Докл. АН СССР, 301, №5, 1037-1040 (1988).

2. Диксмъе Ж. Универсальные обертывающие алгебры. Мир, М., 1978.

3. Элашвили А. Г. Об индексе орисферических подалгебр полупростых алгебр Ли. Труды Тбилисск. матем. ин-та им. Размадзе, 77, 116-126 (1985).

4. Элашвили А. Г. Канонический вид и стационарные подалгебры точек общего положения для простых линейных групп Ли. Функц. анализ и его прил., 6, вып. 1, 51-62 (1972).

5. Charbonnel J.-Y. Propriétés (Q) and (C). Variété commutante, Bull. Soc. Math. France, 132, No. 4, 477-508 (2004).

6. Guralnick R. M. A note on commuting pairs of matrices, Linear and Multilinear Algebra, 31, 71-75 (1992).

7. Neubauer M. G., Sethuraman B. A. Commuting pairs in the centralizers of 2-regular matrices. J. Algebra, 214, No. 1, 174-181 (1999).

8. Panyushev D. Inductive formulas for the index of seaweed Lie algebras. Mosc. Math. J., 1, No. 2, 221-241 (2001).

9. Panyushev D. The index of a Lie algebra, the centralizer of a nilpotent element, and the normalizer of the centralizer. Math. Proc. Cambr. Phil. Soc., 134, No. 1, 41-59 (2003).

10. Panyushev D. Some amazing properties of spherical nilpotent orbits. Math. Z., 245, No. 3, 557-580 (2003).

11. Panyushev D. An extension of Raïs' theorem and seaweed subalgebras of simple Lie algebras. Ann. Inst. Fourier, 55, No. 3, 693-715 (2005).

12. Rosenlicht M. A remark on quotient spaces. An. Acad. Brasil. Cienc., 35, 487-489 (1963).

13. Tauvel P., Yu R. W. T. Indice et formes linéaires stables dans les algèbres de Lie. J. Algebra, 273, No. 2, 507-516 (2004).

Независимый московский университет e-mail: yakimova@mccme.ru

Поступило в редакцию 29 июня 2004 г. 